Article

\title{
Extraction Optimization of Astragaloside IV by Response Surface Methodology and Evaluation of Its Stability during Sterilization and Storage
}

\author{
Lin $\mathrm{Xu}{ }^{1}{ }^{1}$, Kongjiong Wei ${ }^{2}$, Jiaolong Jiang ${ }^{2}$ and Lianfu Zhang ${ }^{1, *}$ \\ 1 School of Food Science and Technology, Jiangnan University, Wuxi 214122, China; linxu7813@gmail.com \\ 2 Gansu Longcuitang Nutrition Food Corp., Ltd., Lanzhou 730046, China; lct_kongjiong@163.com (K.W.); \\ lct_jiaolong@163.com (J.J.) \\ * Correspondence: lianfu@jiangnan.edu.cn; Tel.: +86-510-85917081
}

Citation: Xu, L.; Wei, K.; Jiang, J.; Zhang, L. Extraction Optimization of Astragaloside IV by Response Surface Methodology and Evaluation of Its Stability during Sterilization and Storage. Molecules 2021, 26, 2400. https://doi.org/10.3390/ molecules 26082400

Academic Editor: Francesco Cacciola

Received: 16 March 2021

Accepted: 14 April 2021

Published: 20 April 2021

Publisher's Note: MDPI stays neutral with regard to jurisdictional claims in published maps and institutional affiliations.

Copyright: (c) 2021 by the authors. Licensee MDPI, Basel, Switzerland. This article is an open access article distributed under the terms and conditions of the Creative Commons Attribution (CC BY) license (https:// creativecommons.org/licenses/by/ $4.0 /)$.

\begin{abstract}
Radix Astragali is referred to as a variety of food-medicine herb, and it is commonly applied as Traditional Chinese Medicine (TCM). However, it is extremely difficult to extract its bio-active compounds (astragaloside IV) and apply it in food processing efficiently, which restricts its practical applications. In this study, the conditions required for the extraction of astragaloside IV were optimized by following the response surface methodology. More specifically, ammonia with a concentration of $24 \%$ was used as an extracting solvent, the solid-liquid ratio was 1:10 (w:v); the Radix Astragali was soaked at $25^{\circ} \mathrm{C}$ for $120 \mathrm{~min}$ in advance and then stirred at $25^{\circ} \mathrm{C}$ for $52 \mathrm{~min}(150 \mathrm{rpm})$ to extract astragaloside IV. This method promoted the transformation of other astragalosides into astragaloside IV and replaced the traditional approach for extraction, the solvent reflux extraction method. The yield of astragaloside IV reached the range of $2.621 \pm 0.019 \mathrm{mg} / \mathrm{g}$. In addition, the stability of astragaloside IV was evaluated by detecting its retention rate during sterilization and 60-day storage. As suggested by the results, the astragaloside IV in acidic, low-acidic, and neutral solutions was maintained above $90 \%$ after sterilization $\left(95^{\circ} \mathrm{C}\right.$ and $60 \mathrm{~min}$ ) but below $60 \%$ in an alkaline solution. High temperature and short-term sterilization approach is more appropriate for astragaloside IV in an alkaline solution. It was also found out that the astragaloside IV obtained using our method was maintained over $90 \%$ when stored at room temperature $\left(25^{\circ} \mathrm{C}\right)$, and there was no significant difference observed to low temperature $\left(4^{\circ} \mathrm{C}\right)$ in solutions regardless of acidity.
\end{abstract}

Keywords: astragaloside IV; response surface methodology; stability; heat sterilization; storage

\section{Introduction}

Radix Astragali (Huangqi), a widely-known traditional food-medicine herb [1], has been approved to be applied as food ingredients by the National Health Commission of China and the European Food Safety Authority (EFSA) Panel on Nutrition [2]. However, the most common application of Radix Astragali is water-soaking [3-5], without considering efficiency and the stability of the main bioactive compound [6], astragaloside IV. Astragaloside IV is considered the herb's major bioactive component [7] for its pharmacological and pharmacokinetic properties, such as its anti-inflammatory effect [8], anticancer activity [9], and neuroprotective activity [10]. At present, astragaloside IV is recognized by the Chinese Pharmacopoeia (2015 version) as a qualitative control standard. However, astragaloside IV is disadvantaged by a poor solubility (both in water and organic solvents) and low content, which makes the extraction of it far from efficient [11]. Additionally, it is possible for other astragalus saponins in Radix Astragali, especially astragaloside I and astragaloside II [12], to be transformed into astragaloside IV in certain conditions. The chemical structures of astragaloside I, II, and IV were shown in Figure 1. The addition of lye into the solvent can help hydrolyze the acetyl base to fall off on the xylan terminal chain 
and convert other astragalus saponins into astragaloside IV easily [13], thus improving the yield of astragaloside IV.

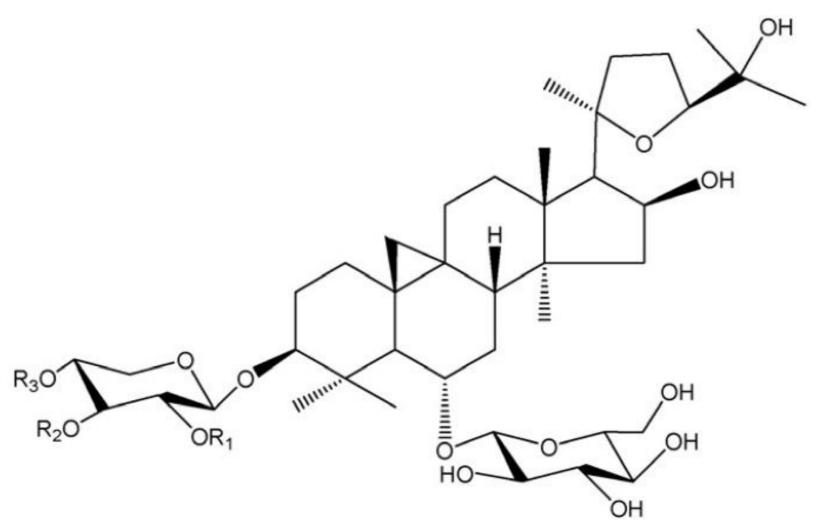

Figure 1. The chemical structures of astragaloside I, II, and IV [14]. Astragaloside I: R1 = R2 = Ac, $\mathrm{R} 3=\mathrm{H}$; astragaloside II: R1 = Ac, R2 = R3 = H; astragaloside IV: R1 = R2 = R3 = H.

There are various influencing factors in the transformation of other astragalus saponins into astragaloside IV, e.g., the type and concentration of the alkaline solution, the solidliquid ratio, the soaking time, the extraction time, and the extraction temperature. On the one hand, too much alkaline and a too-high extraction temperature can cause the saponin structure to collapse and reduce the yield. Mei Xiaodan et al. [15] explored the yield of astragaloside IV extracted for 30 min using a 2.5-25\% ammonia and 1\% sodium hydroxide solution, but this failed to achieve any further optimization. The problem lies in how to remove the alkaline in a non-toxic way. In some other studies [12,15], a macroporous resin elution was used to remove alkaline after extraction. However, this method consumed too much energy and was toxic due to the risk of particle residue. Thus, this method is unsuited to food processing. Therefore, it is essential to identify suitable alkaline species and determine favorable extraction conditions.

In addition, the instability of the astragaloside IV extract, especially the alkaline extract, is an urgent problem to be solved. Firstly, astragaloside IV is a natural extract, and the stability of natural extracts is a matter of great concern. Because the instability of natural extracts can make products unstable and cause curative effects, it is difficult to provide stable products for consumers. The content of astragaloside is easily affected by temperature and acidity, but the effect is not clear. Secondly, as a new resource food raw material, there is little research on the stability of Radix Astragali or astragaloside IV in food processing. Zheng Na et al. [16] researched the retention rate of astragaloside I, II, and IV standard in pH 2.0 and 6.8 solutions within $80 \mathrm{~h}$, but these were far from application scenarios. In order to simulate the acidity of the digestive system, Feng Xiaoquan et al. [17] conducted an investigation into the content of the astragaloside IV in a $0.1 \mathrm{~mol} \cdot \mathrm{L}^{-1} \mathrm{HCl}$ solution and $8 \%$ ammonia, which led to the finding that the content of astragaloside IV is affected by acidity. Additionally, these limited studies were based on the astragaloside standard rather than the extract system. Thirdly, exploring the stability of an extract obtained using this method can provide guidance on the subsequent processing of Radix Astragali to some extent, e.g., the improvement of sterilization or storage conditions. According to the level of acidity, food is categorized into acidic, low-acidic, neutral, and alkaline foods, with different types of food requiring different sterilization conditions [18]. It is thus necessary to investigate the change in the retention rate of astragaloside IV after lye extraction under different conditions of sterilization processing and storage, which will contribute not only to ensuring high quality and safe products for consumers but also to widening the scope of applications for Radix Astragali. In this paper, the relationship between the thermal sterilization stability and acidity of astragaloside IV was investigated, and suggestions were put forward to ensure the stability of astragaloside IV in processing. 
Moreover, there is still no research on the retention rate of astragaloside IV extracted by lye after sterilization during storage.

Different from other studies $[12,15]$, this study features the introduction of the pretreatment process before the extraction of astragaloside IV, which shortens the extraction time, reduces energy consumption, and improves extraction efficiency. Additionally, an ammonia solution is taken as the solvent, because it is easy to remove by vacuum evaporation and the need to use macroporous resin elution methods (which carries the risk of macroporous resin residue and is prohibited from food processing) is avoided. The response surface method is frequently used to analyze multiple variables responding to one or more responses $[19,20]$. In this study, the extraction conditions were optimized for the astragaloside IV by following the response surface methodology. At the same time, an exploration was conducted into the stability of astragaloside IV during the common sterilization processes in acidic, low-acidic, neutral, and alkaline conditions, as well as in 60-day storage. In addition to the yield of astragaloside IV, astragaloside I and astragaloside II were also detected by ultra-performance liquid chromatography-tandem mass spectrometry (UPLC-MS/MS) [21] throughout the process (extraction, sterilization, and storage) in order to establish whether the transformation of astragaloside IV would be reversed.

\section{Materials and Methods}

\subsection{Materials}

Radix Astragali was collected from Longxi, Gansu Province in China. After being cleaned and dried in a heating-air drying oven at about $40{ }^{\circ} \mathrm{C}$ until constant weight, the raw materials were smashed and sieved through a 6-mesh sieve. The powder was sealed in a PE bag and then kept at $-20^{\circ} \mathrm{C}$ for later use. Astragaloside I, astragaloside II, and astragaloside IV standard compounds were purchased from Shanghai yuan ye Bio-Technology Co., Ltd. (Shanghai, China) with a purity of $>98 \%$. Methanol (HPLC grade), acetonitrile (HPLC grade), methanoic acid (HPLC grade), ammonium hydroxide, and ethanol were sourced from Sinopharm Chemical Reagent Co., Ltd. (Shanghai, China). Ultrapure water was used for the preparation of all solutions.

\subsection{Quantitation of Astragaloside I, Astragaloside II, and Astragaloside IV}

The quantitation of astragaloside was performed using the method proposed by Qi L.W. et al. [14] with some modifications. UPLC-MS/MS quantitated all astragalosides with a UPLC-TQD system in the + ESI mode. The purified sample $(1 \mu \mathrm{L})$ was injected into an ACQUITY C18 $(1.7 \mu \mathrm{m}, 2.1 \times 100 \mathrm{~mm})$ column with a gradient elution comprised of eluents A $(100 \%$ acetonitrile) and B $(0.1 \%, v / v$ formic acid in water) at $0.3 \mathrm{~mL} / \mathrm{min}$. The column temperature was maintained at $45^{\circ} \mathrm{C}$, and the mobile phase elution procedure was as follows: $0-1 \mathrm{~min}, 20 \% \mathrm{~A} ; 1-10 \mathrm{~min}, 20-100 \% \mathrm{~A} ; 10-11 \mathrm{~min}, 100 \% \mathrm{~A} ; 11-12 \mathrm{~min}, 100-20 \%$ $\mathrm{A}$; and $12-14 \mathrm{~min}, 20 \% \mathrm{~A}$. The MS/MS analysis was conducted at 700-900 m/z, with N2 ( $>99.99 \%$ ) as the drying gas and the flow set to $500 \mathrm{~L} / \mathrm{h}$. The source block temperature and desolvation temperature were 100 and $400{ }^{\circ} \mathrm{C}$, respectively. The capillary voltage was set to $3.0 \mathrm{kV}$, and the detector voltage was set to $1800 \mathrm{~V}$. Mass Lynx software was applied for data and graph analysis. The quantitation of astragalosides was performed using a calibration curve of the corresponding purified astragalosides.

\subsection{Quantitative Validation Calibration Curves}

\subsubsection{Calibration Curves, Limits of Detection (LOD) and Quantification (LOQ)}

In order to plot the calibration curves, methanol stock solutions containing astragaloside I, II, and IV were prepared and diluted to appropriate ranges of concentration. The quantitation of astragaloside I, astragaloside II, and astragaloside was identified following Section 2.2, while the linear regression equations of astragaloside I, II, and IV were calculated with the mass concentration of astragaloside I, II, and IV as the horizontal coordinate and the peak area as the vertical coordinate. Under the present chromatographic condi- 
tions, the LOD and LOQ were determined at the signal-to-noise ratios (S/Ns) of 3 and 10 , respectively.

\subsubsection{Precision and Accuracy}

With $4 \mathrm{~g}$ of sample accurately weighed, the sample solution was prepared following Section 2.4., while the retention time and peak area of each substance were determined 6 consecutive times according to Section 2.2. within the space of $1 \mathrm{~d}$. The intra-day precision was calculated using relative standard deviation (RSD\%). Similarly, the same process was repeated 6 times per day for 3 consecutive days in order to calculate the inter-day precision by using RSD\%. Additionally, $1 \mathrm{~g}$ of the samples with a known content of astragaloside I, II, and IV was accurately weighed, and this was repeated 6 times. With astragaloside I, II, and IV at concentrations of $26.67,13.33,8.33 \mu \mathrm{g} / \mathrm{mL}$, respectively, $1 \mathrm{~mL}$ of a standard solution was added. The sample solution was prepared according to Section 2.4., the content of each substance was determined according to Section 2.2., and the recoveries were calculated $(\%)$.

\subsection{Single-Factor Experiments of the Extraction Process}

The control variable method was adopted to investigate the potential impact of ammonia concentration, solid-liquid ratio, soaking time, extraction time, extraction temperature, and extraction time on the yield of astragaloside IV, with the separation of astragaloside I, II, and IV performed in accordance with the work of Shaw et al. PLoS one, Published: 29 August 2012, https:/ / doi.org/10.1371/journal.pone.0043848 [22]. To be specific, about $3 \mathrm{~g}$ of astragalus powder were accurately weighed, while a certain volume of an ammonia solution of a certain concentration was added and soaked at $25^{\circ} \mathrm{C}$ for some time. Additionally, an oscillating extractor was used at the given temperature at $180 \mathrm{rpm}$ for some time. Then, the solution was concentrated by vacuum-rotary evaporation at $45^{\circ} \mathrm{C}$. The residue was dissolved by $10 \mathrm{~mL}$ of water and extracted by $40 \mathrm{~mL}$ of n-butanol saturated with water. After extraction was performed four times, the extraction of n-butanol saturated with water was combined. The solution was concentrated to $10 \mathrm{~mL}$ by vacuum-rotary evaporation at $45^{\circ} \mathrm{C}$ and filtered using a filter $(0.22 \mathrm{~lm}$; Whatman, Maidstone, UK), suitable for UPLCTQD analysis. The astragaloside IV was determined, and the yield was calculated using Equation (1), with the analysis of parallel measurements done three times:

The astragaloside IV yield = Weight of astragaloside IV (mg)/Weight of Radix Astragali (g)

The specific extraction conditions were set as follows:

\subsubsection{Ammonia Concentration}

The ammonia concentration was set to $0 \%, 10 \%, 20 \%, 30 \%$, and $40 \%$. The solidliquid ratio, soaking time, extraction time, extraction temperature, and number of times of extraction were set to $1: 10,30 \mathrm{~min}, 30 \mathrm{~min}, 20^{\circ} \mathrm{C}$, and 1, respectively.

\subsubsection{Solid-Liquid Ratio}

The solid-liquid ratio was set to 1:3, 1:10, 1:20, 1:30, and 1:40. The ammonia concentration, soaking time, extraction time, extraction temperature, and number of times of extraction were set to $20 \%, 30 \mathrm{~min}, 30 \mathrm{~min}, 20^{\circ} \mathrm{C}$, and 1 , respectively.

\subsubsection{Soaking Time}

The soaking time was set to $0,60,120,180$, and $240 \mathrm{~min}$. The ammonia concentration, solid-liquid ratio, extraction time, extraction temperature, and number of times of extraction were set to $20 \%, 1: 10,30 \mathrm{~min}, 20^{\circ} \mathrm{C}$, and 1, respectively. 


\subsubsection{Extraction Time}

The extraction time was set to $0,60,120,180$, and $240 \mathrm{~min}$. The ammonia concentration, solid-liquid ratio, soaking time, extraction temperature, and number of times of extraction were set to $20 \%, 1: 10,60 \mathrm{~min}, 20^{\circ} \mathrm{C}$, and 1 , respectively.

\subsubsection{Extraction Temperature}

The extraction temperature was set to $0,20,40,60$, and $80^{\circ} \mathrm{C}$. The ammonia concentration, solid-liquid ratio, soaking time, extraction time, and number of times of extraction were set to $20 \%, 1: 10,60 \mathrm{~min}, 30 \mathrm{~min}$, and 1, respectively.

\subsubsection{Extraction Times}

After the first-time extraction, the solid was recycled and extracted a second time and a third time. The ammonia concentration, solid-liquid ratio, soaking time, extraction time, and extraction temperature were set to $20 \%, 1: 10,60 \mathrm{~min}, 30 \mathrm{~min}$, and $20^{\circ} \mathrm{C}$, respectively.

\subsection{Response Surface Method to Optimize Extraction Conditions}

From the single-factor experiments conducted on the extraction process, it was known that the extraction temperature caused a reduction to the yield of astragaloside. The number of times of extraction times made no significant difference to the yield of astragaloside. Therefore, ammonia concentration (A), solid-liquid ratio (B), soaking time (C), and extraction time (D) were selected as the factors to investigate, while the yield of astragaloside IV was treated as the investigative indicator. Additionally, the Box-Behnken approach was used to design a four-factor and three-level response surface experiment. Table 1 shows the factors and levels. The solution was concentrated to $10 \mathrm{~mL}$ by means of vacuum-rotary evaporation and filtered using a filter $(0.22 \mathrm{~lm}$; Whatman, Maidstone, UK) for subsequent UPLC-TQD analysis. The optimization conditions were predicted using a second-order polynomial equation.

Table 1. The factors and levels for the response surface methodology design.

\begin{tabular}{cccc}
\hline The Factors & \multicolumn{3}{c}{ Level } \\
\cline { 2 - 4 } & $\mathbf{- 1}$ & $\mathbf{0}$ & $\mathbf{1}$ \\
\hline Ammonia concentration (A) $(\%, v / v)$ & 20 & 25 & 30 \\
Solid-liquid ratio (B) $(\mathrm{mL} / \mathrm{g})$ & $10: 1$ & $15: 1$ & $20: 1$ \\
Soaking time (C) (min) & 60 & 90 & 120 \\
Extraction time (D) (min) & 0 & 30 & 60 \\
\hline
\end{tabular}

\subsection{The Retention Rate of the Astragaloside IV Under Different Sterilization Conditions}

According to the conditions required for response surface optimization, the extract of astragaloside IV was obtained at a $24.15 \%$ ammonia concentration, a solid-liquid ratio of 1:10, a soaking time of $120 \mathrm{~min}$, and an extraction time of $52 \mathrm{~min}$. The $\mathrm{pH}$ value of the solution at 3,5,7, and 9 was adjusted to simulate acidic, low-acidic, neutral, and alkaline food environments, respectively. The solution was simulated at a given sterilization temperature and time. The treated solution was treated using the methods in Section 2.2 so as to detect the changes of astragaloside I, astragaloside II, and astragaloside IV in the solution. The specific sterilization conditions are shown as follows: heating at $85^{\circ} \mathrm{C}$ for 30 , 40,50 , and $60 \mathrm{~min}$; heating at $90^{\circ} \mathrm{C}$ for $30,40,50$, and $60 \mathrm{~min}$; heating at $95^{\circ} \mathrm{C}$ for $30,40,50$, and $60 \mathrm{~min}$; heating at $85^{\circ} \mathrm{C}$ for $30,40,50$, and $60 \mathrm{~min}$; heating at $100^{\circ} \mathrm{C}$ for 15 and $30 \mathrm{~min}$; heating at $105^{\circ} \mathrm{C}$ for $15 \mathrm{~min}$; and heating at $120^{\circ} \mathrm{C}$ for $10 \mathrm{~min}$. The astragaloside IV content was determined by UPLC-TQD, the retention rate was calculated using Equation (2), and parallel measurements were analyzed three times: 
2.7. The Retention Rate of Astragaloside IV in Different $p H$ Value Solutions Stored at 4 or $25^{\circ} \mathrm{C}$

After sterilization, solutions with different $\mathrm{pH}$ values were vacuum-sealed in aluminum foil packaging bags and then stored at 4 and $25^{\circ} \mathrm{C}$ for 60 days, which is common during the transportation and storage of food and beverages [23]. During the period, the content of astragaloside IV was detected to calculate the retention rate through regular inspection. Moreover, the contents of astragaloside I and astragaloside II were detected to determine whether the transformation of astragaloside IV was reversed.

\subsection{Statistical Analysis}

A response surface data analysis was conducted using the Design Expert. V8.0.6 (Minneapolis, MI, USA) software, and other statistical data analyses were carried out using GraphPad Prism 8.0.2 (GraphPad Software Inc., San Diego, CA, USA). In addition, a $t$-test was performed to make unpaired observations, based on which the statistical evaluation of differences was conducted, with a $p$-value less than 0.05 treated as statistically significant. All tests were carried out through three parallel operations, with the results expressed as mean \pm SD.

\section{Results and Discussion}

3.1. Identification of Astragaloside I, Astragaloside II, and Astragaloside IV by UPLC-MS/MS

Figure 2 shows the UPLC-MS/MS chromatogram of astragaloside I, astragaloside II, and astragaloside IV. Table 2 lists the MRM parameters of astragaloside I, astragaloside II, and astragaloside IV.

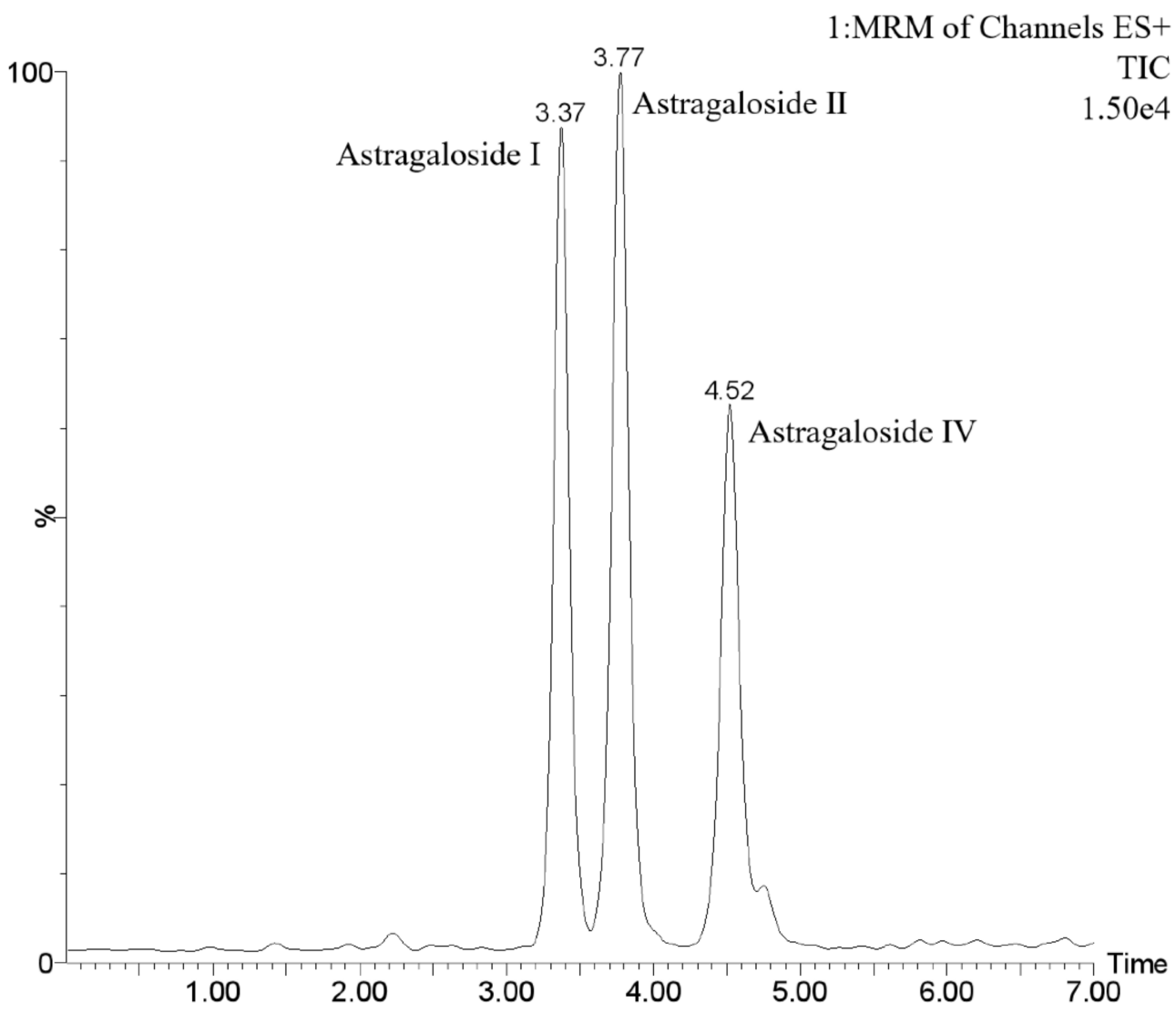

Figure 2. The UPLC-TQD chromatogram of astragaloside I, astragaloside II, and astragaloside IV. 
Table 2. MRM parameters of astragaloside IV.

\begin{tabular}{ccccc}
\hline & Qualitative Ion Pair & Quantitative Ion Pair & Cone Hole Voltage/V & Collision Energy/eV \\
\hline Astragaloside I & $891.5>891.5$ & $891.5>891.5$ & 13 & 10 \\
\hline \multirow{3}{*}{ Astragaloside II } & $849.4>849.4$ & & 14 & 9 \\
& $827.5>827.5$ & $849.4>849.4$ & 14 & 10 \\
\hline \multirow{2}{*}{ Astragaloside IV } & $827.5>143$ & & 14 & 11 \\
\hline
\end{tabular}

\section{Quantitative Validation}

As shown in Table 3, the linearity of analytical response was acceptable with the correlation coefficients higher than 0.99 , thus providing a dynamic range of about two orders of magnitude. The LODs of astragaloside I, astragaloside II, and astragaloside IV fell within the range of $0.002-0.006 \mu \mathrm{g}$, the LOQs varied from 0.009 to $0.024 \mu \mathrm{g}$, the intra-day RSDs ranged between $1.4 \%$ and 3.7\%, the inter-day RSDs varied between $1.1 \%$ and $1.8 \%$, and the recoveries exceeded $94 \%$. Suitable for the detection of astragaloside I, astragaloside II, and astragaloside IV, this method is effective in shortening the detection time and improving efficiency.

Table 3. Quantitative validation.

\begin{tabular}{cccc}
\hline \multirow{2}{*}{ Parameters } & \multicolumn{3}{c}{ Substances } \\
\cline { 2 - 4 } & Astragaloside I & Astragaloside II & Astragaloside IV \\
\hline Calibration curve & $\mathrm{y}=400.12 \mathrm{x}+1309.5$ & $\mathrm{y}=655.11 \mathrm{x}+1353.9$ & $\mathrm{y}=1047.7 \mathrm{x}+197.46$ \\
Test range $(\mu \mathrm{g} / \mathrm{mL})$ & $0.083-2.67$ & $0.042-1.33$ & $0.026-0.83$ \\
$\mathrm{r}^{2}$ & 0.9991 & 0.9935 & 0.9997 \\
LOD $(\mu \mathrm{g})$ & 0.006 & 0.003 & 0.002 \\
LOQ $(\mu \mathrm{g})$ & 0.024 & 0.015 & 0.009 \\
Intra-day RSD $(\%)(n=5)$ & 1.5 & 3.7 & 1.4 \\
Inter-day RSD $(\%)(n=5)$ & 1.8 & 1.2 & $96.5 \pm 1.9$ \\
Recoveries $(\%)$ & $94.1 \pm 4.3$ & $98.4 \pm 5.8$ & 96.1 \\
\hline
\end{tabular}

y: peak area ratio of the analyte/internal standard; $x$ : concentration of analyte $(\mu \mathrm{g} / \mathrm{mL})$.

\subsection{Single-Factor Experiments}

\subsubsection{Ammonia Concentration}

Ammonia concentration is one of the most critical influencing factors in the content and yield of saponins. As shown in Figure 3a, the yield of astragaloside IV first increased and then decreased when the ammonia concentration varied between $0 \%$ and $40 \%$. In comparison, higher and lower ammonia concentrations caused reductions to the content and yield of saponins. That is to say, an insufficient alkali content will lead to an incomplete conversion between astragalus saponins, but an excessive amount of ammonia is suitable to hydrolyze astragalus saponins [15]. According to the results, the ammonia concentration was set to the range of $20-30 \%$ for extracting the astragalus saponins.

\subsubsection{Solid-Liquid Ratio}

Increasing the liquid-to-solid ratio is an effective solution to improving the content and yield of astragaloside IV by adjusting the concentration gradient in and out of cells in the solution. In this study, the water-to-solid ratios of 3, 10, 20, 30, and 40 were investigated. As shown in Figure 3b, as the water-to-solid ratio rose sharply from 3 to 20, and the content and yield of astragaloside IV increased accordingly, which was possibly because the rising water-to-solid ratio increased the diffusion of solvent in cells and enhanced the desorption of astragaloside IV from cells [24]. When the water-to-solid ratios were set at 30 and 40, however, the components were completely dissolved into the extract. As a result, the variation in the yield of astragaloside IV was found to be insignificant $(p<0.05)$. Thus, the water-to-solid ratio was set to $10-20 \%$ for the extraction of astragalus saponins. 


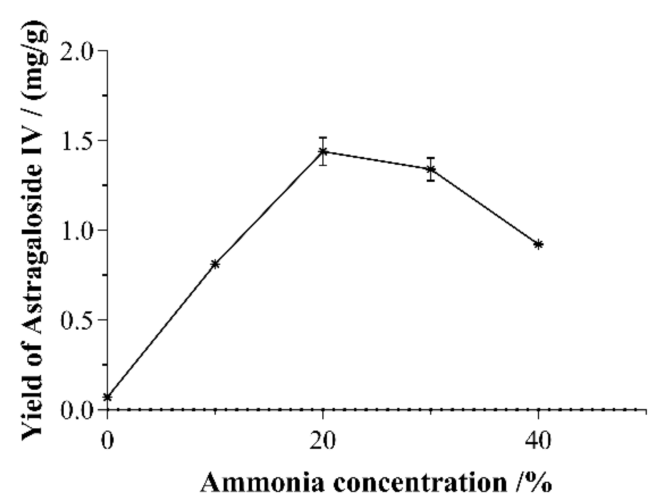

(a)

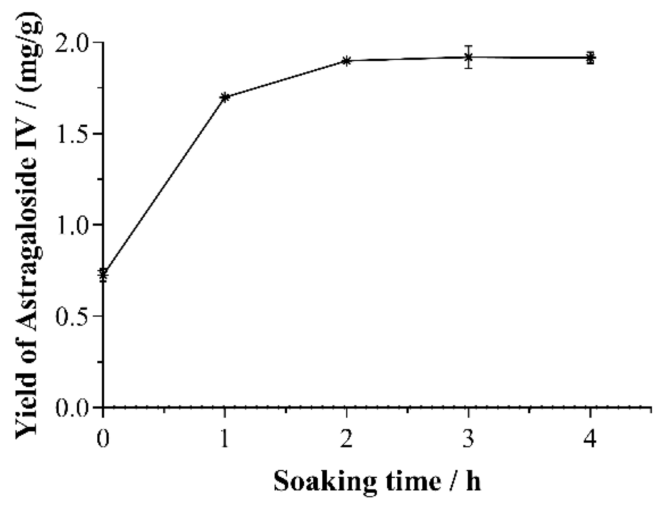

(c)

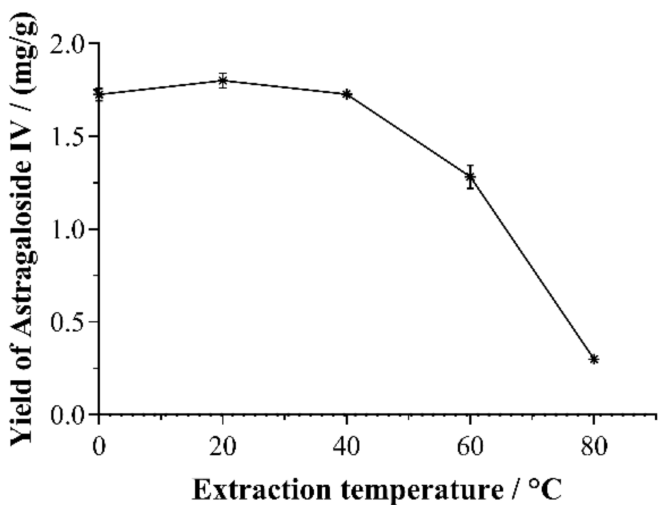

(e)

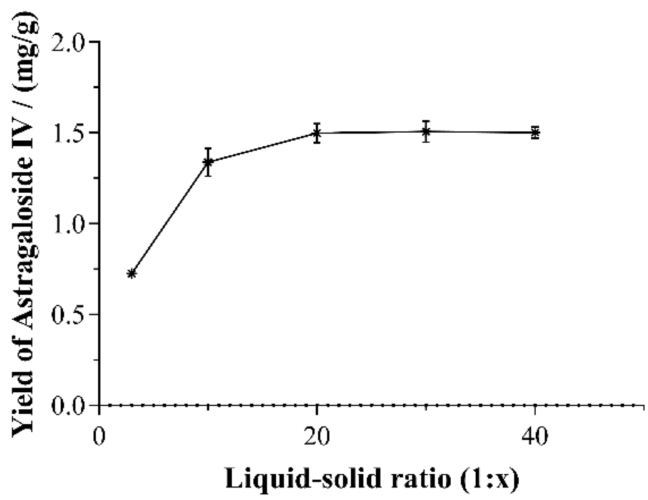

(b)

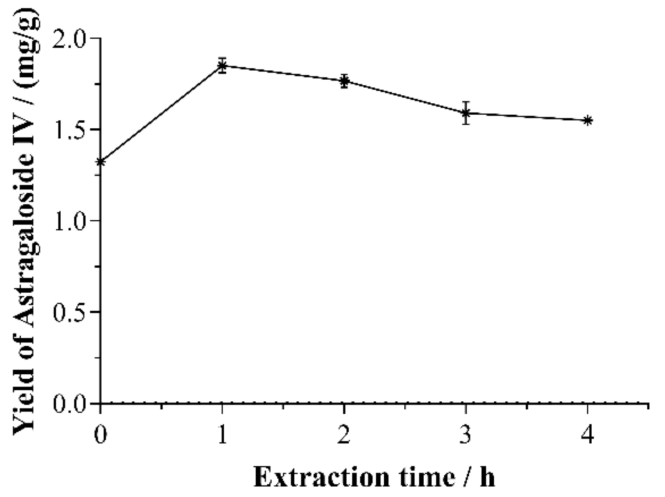

(d)

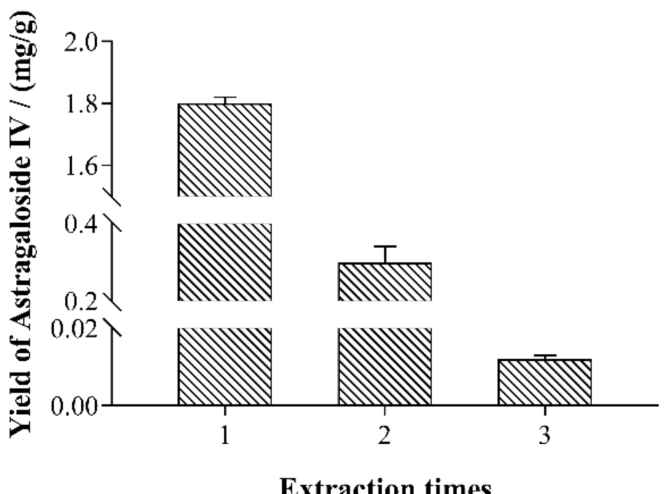

(f)

Figure 3. The effects of ammonia concentration (a), solid-liquid ratio (b), soaking time (c), extraction time (d), extraction temperature (e), and extraction time (f) on the yield of astragaloside IV in single-factor experiments of the extraction process.

\subsubsection{Soaking Time}

Then, an investigation was conducted into the impact of soaking time on the yield of astragaloside IV, with the extraction time sets to $0,1,2,3$, and $4 \mathrm{~h}$. According to Figure $3 c$, as the soaking time was extended, the yield of astragalus saponins first increased rapidly and then slowed down. This was because a longer soaking time was conducive to the transformation of astragalus saponins at the initial stage of soaking [15]. Other astragalus saponins could be transformed into astragaloside IV immediately. With the further extension to the soaking time, the transformation process got close to completion, 
and the effect of increasing the yield of astragaloside IV was made less significant than before. In order to reduce cost, the soaking time was determined as 60-120 $\mathrm{min}$ in the following response surface methodology experiments.

\subsubsection{Extraction Time}

The effects of the extraction time on the yield of astragalus saponins were then investigated with the extraction times set to $0,1,2,3$, and $4 \mathrm{~h}$. As shown in Figure 3d, the yield of astragalus saponins first increased and then declined as the extraction process was prolonged. The reason behind it was that, at the initial stage of extraction, a longer extraction time was favorable to dissolving astragaloside IV and other polar compounds in the solvent. However, with an extension to this period, it was easy for astragaloside $\mathrm{IV}$, as a kind of triterpenoid saponins, to be decomposed [25]. The concentration of other ingredients in the solvent reached the equilibrium, so the change of dissolution was limited. Additionally, there might have been some adverse reactions taking place that reduced the yield. Additionally, over the course of extraction, the increase of suspension viscosity was unfavorable to improving the extraction efficiency, and astragaloside IV could hardly be extracted from cell debris [26]. Based on the results, the extraction lasting $0-1 \mathrm{~h}$ was judged as effective in achieving a higher yield of saponins. Therefore, $0,0.5$, and $2 \mathrm{~h}$ were treated as the optimal conditions in the following response surface methodology experiments.

\subsubsection{Extraction Temperature}

On the one hand, increasing the extraction temperature can improve the efficiency of transformation for astragalosides. On the other hand, a high temperature could accelerate the collapse of astragaloside IV [15,25]. As shown in Figure 3e, the yield of astragaloside IV slowly increased with the rise of extraction temperature up until $40^{\circ} \mathrm{C}$ and then decreased. In order to reduce costs, the extraction temperature was determined as $20^{\circ} \mathrm{C}$.

\subsubsection{Extraction Times}

Figure $3 \mathrm{f}$ shows the impact made by the number of times of extraction on the yield of astragaloside IV. It could be seen from the figure that the second-time extraction led to as little as $0.326 \pm 0.017 \mathrm{mg}$ being extracted. This was due to the presence of some astragaloside residues in the gap of astragalus powder during the extraction process. Additionally, production costs were continuously on the rise. Therefore, the number of times of extraction was set to two as the optimal condition in the following experiments. Moreover, for the second-time extraction, the liquid-to-solid ratio was set to $1: 1$ for cost reduction.

\subsection{Response Surface Methodology}

\subsubsection{Response Surface Experimental Design and Response}

As shown in Tables 1 and A1, there were twenty-nine tests conducted under different conditions, as designed using the response surface method. Moreover, Table A1 lists the experimental results corresponding to each test combination. The yields of astragaloside I and astragaloside II were also detected at the same time as astragaloside IV, as shown in Appendix A,Table A1. Moreover, Design-Expert, version 8.6 (Stat-Ease Inc., Minneapolis, MN, USA) was applied to calculate the multiple linear regression equation according to the response value and the experimental conditions. The software was also used to calculate the regression coefficients. The fitted equations to predict the yield of astragaloside IV from the Radix astragali are given as follows:

The yield of astragaloside IV $=2.19-0.36 \times \mathrm{A}+8.167 \times 10^{-3} \times \mathrm{B}+0.26 \times \mathrm{C}+0.091 \times \mathrm{D}-0.013$

$\times \mathrm{A} \times \mathrm{B}+0.085 \times \mathrm{A} \times \mathrm{C}-0.040 \times \mathrm{A} \times \mathrm{D}-0.12 \times \mathrm{B} \times \mathrm{C}+0.038 \times \mathrm{B} \times \mathrm{D}+0.045 \times \mathrm{C} \times \mathrm{D}-0.86$

$$
\times \mathrm{A}^{2}-0.012 \times \mathrm{B}^{2}+0.038 \times \mathrm{C}^{2}-0.069 \times \mathrm{D}^{2}
$$

According to the results shown in Table A1, astragaloside I and astragaloside II were not detected in most test combinations, suggesting that the transformation of astragaloside I and astragaloside II into astragaloside IV was complete in the corresponding tests. As 
revealed by an analysis of the experimental results, the yield of astragaloside IV obtained after alkaline washing was slightly higher than the sum of astragaloside I, astragaloside II, and astragaloside IV. These results also demonstrated that saponins other than astragaloside I and astragaloside II in Radix Astragali could also be converted into astragaloside IV, as suggested in other studies [12,13].

\subsubsection{The ANOVA of Response Surface Methodology}

ANOVA in the software was conducted to evaluate the quadratic polynomial models [26]. A larger F-value and smaller $p$-value imply a more significant effect on the respective response methodology [27]. Table 4 lists the ANOVA results obtained for some important terms of the models. The adequacy of the model could be verified against the coefficient of determination $\left(\mathrm{R}^{2}\right)$, the lack of fit, $\mathrm{R}^{2}$ adj, $\mathrm{AP}$, and $\mathrm{CV}$ tests. Among these parameters, $\mathrm{R}^{2}$ denotes the percentage of the variables as can be explained using the model. A higher $\mathrm{R}^{2}$ represents most of the variables, which can be explained by the model. The experimental data were consistent with the second-order polynomial equation. As for the parameters shown in Table 4 , the $\mathrm{R}^{2}$ value was 0.9927 . That is to say, the total variation reached only $0.73 \%$, which could not be explained by the model. In this model, the value of $R^{2}$ was sufficiently high to meet our requirement. In the meantime, $R^{2}$ adj is as authoritative as $R^{2}$ for the parameter $R^{2}$ adj which is a modification of $R^{2}$, with adjustment made to the number of descriptive terms. Different from $R^{2}, R^{2}$ adj only increases when the new term improves the model more significantly than expected [28]. It is better if the values of $R^{2}$ and $\mathrm{R}^{2}$ adj are greater and closer to each other. From Table 4 , it can be seen that the value of $R^{2}$ adj of the model was 0.9853 . The high value of $R^{2}$ adj indicated that the model was significant. As indicated by the significance shown by the lack of fit test, the points were not adequately distributed around the model. Thus, the model could not be applied to predict the values of the independent variables. Therefore, the insignificance shown by the lack of fit test implied that the model could fit the data well [11]. In our model, all the " $p$-value prob $>\mathrm{F}^{\prime}$ " values of the lack of fit exceeded $10 \%$, and they were insignificant. As indicated by these parameters, the model could fit the results well. AP was used to measure the signal-to-noise ratio, and a ratio greater than 4 was treated as desirable. In this model, the value of AP was 41.691, indicating that the signal was adequate. Moreover, the CV was supposed not to exceed $10 \%$ [29]. The value of the CV in our model was 3.52. In addition to the above-mentioned parameters, the predicted versus measured figure could also be used to demonstrate the suitability of the model. As shown in Figure 4, the predicted and actual measured experiment values were fitted almost in a straight line. As evidenced by this figure, the second-order polynomial regression model fit the experimental results well. Therefore, the models applied in this study could optimize the conditions for extracting astragaloside IV from Radix Astragali.

\subsubsection{The Impact of Different Factors on the Yield of Astragaloside IV}

In Table 4, the linear and quadratic effects of the ammonia concentration, soaking time, and extraction time are shown to be significant $(p<0.05)$. The ammonia concentration with soaking time and solid-liquid ratio with soaking time were also found to be significant $(p<0.05)$.

Figure 5a shows the interaction between the ammonia concentration with soaking time. The yield of astragaloside IV first increased with the concentration of the ammonia and then decreased, which could be attributed to the treatment of alkaline wash that played a role in promoting the transformation of the astragalosides replaced with an acetyl group to astragaloside IV. Additionally, the higher alkalinity, the higher the degree of its transformation within the limits (ammonia concentration $\leq 25 \%$ ). In this process, the production of astragaloside IV was significantly higher than the consumption of it. When alkalinity was sufficient, however, the saponins could also be effectively destroyed [15]. Thus, the yield of astragaloside IV treated with 30\% ammonia was lower as compared to that treated with $25 \%$ ammonia. A longer soaking time provided more opportunities for 
other kinds of saponins to be transformed into astragaloside IV. As shown in Figure 5a,b, the yield of astragaloside IV reached its maximum when the ammonia concentration and soaking time approached $24 \%$ and $120 \mathrm{~min}$, respectively.

Table 4. Results of the ANOVA to the response surface quadratic model for the yield of astragaloside IV.

\begin{tabular}{cccccc}
\hline Content of Astragaloside IV & Sum of Squares & Degrees of Freedom & Mean Square & F-Value & $p$-value \\
\hline Model & 7.71 & 14 & 0.55 & 135.37 & $<0.0001$ \\
A & 1.52 & 1 & 1.52 & 373.33 & $<0.0001$ \\
B & $8.00 \times 10^{-4}$ & 1 & $8.00 \times 10^{-4}$ & 0.20 & 0.6642 \\
C & 0.80 & 1 & 0.80 & 196.77 & $<0.0001$ \\
D & 0.099 & 1 & 0.099 & 24.42 & 0.0002 \\
AB & $6.25 \times 10^{-4}$ & 1 & $0.25 \times 10^{-4}$ & 7.10 & 0.7010 \\
AC & 0.029 & 1 & $6.40 \times 10^{-3}$ & 1.57 & 0.2304 \\
AD & $6.40 \times 10^{-3}$ & 1 & 0.053 & 13.00 & 0.0029 \\
BC & 0.053 & 1 & $5.77 \times 10^{-3}$ & 1.42 & 0.2533 \\
BD & $5.77 \times 10^{-3}$ & 1 & $8.1 \times 10^{-3}$ & 1.99 & 0.1801 \\
CD & $8.1 \times 10^{-3}$ & 1 & 4.81 & 1181.04 & $<0.0001$ \\
A2 & 4.81 & 1 & $9.87 \times 10^{-4}$ & 0.24 & 0.6301 \\
B2 & $9.87 \times 10^{-4}$ & 1 & $9.33 \times 10^{-3}$ & 2.29 \\
C2 & $9.33 \times 10^{-3}$ & 1 & 0.031 & 7.50 \\
D2 & 0.031 & 14 & $4.07 \times 10^{-3}$ & 0.1523 \\
Residual & 0.057 & 10 & $5.25 \times 10^{-3}$ & 4.64 \\
Lack of Fit & 0.052 & 4 & $1.13 \times 10^{-3}$ & 0.0160 \\
Pure Error & $4.52 \times 10^{-3}$ & 28 & & 0.0761 \\
Cor Total & 7.77 & & 0.9927 & &
\end{tabular}

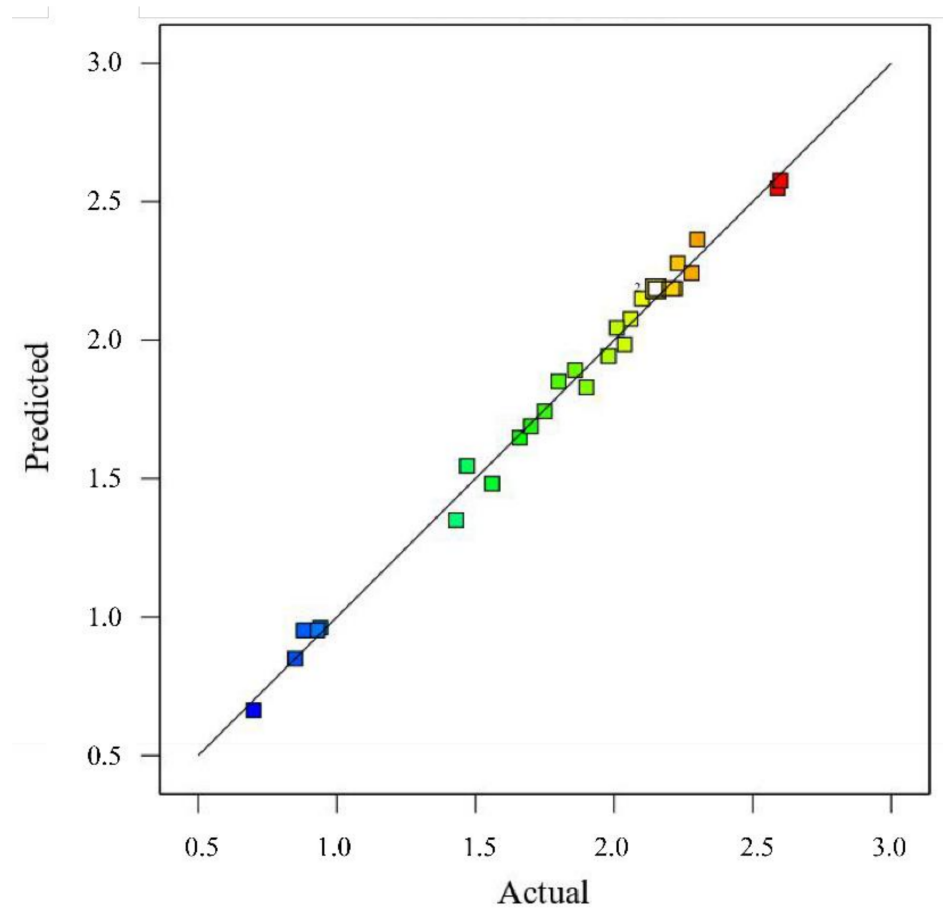

Figure 4. The comparison between the predicted and measured values of the yield of the astragaloside IV from Radix Astragali. 


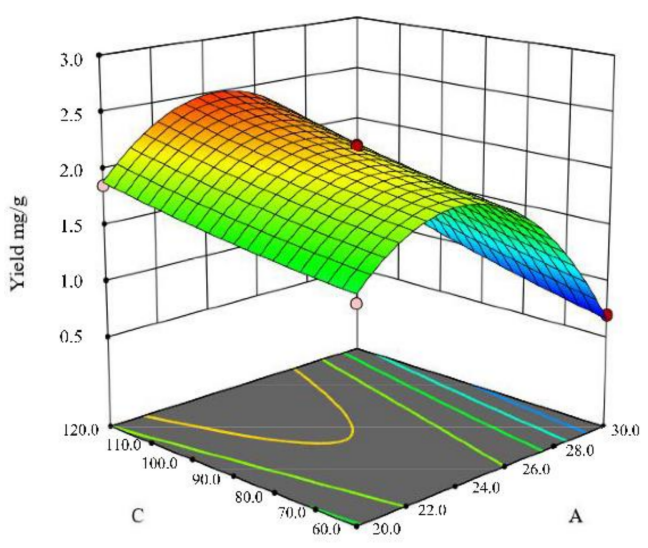

(a)

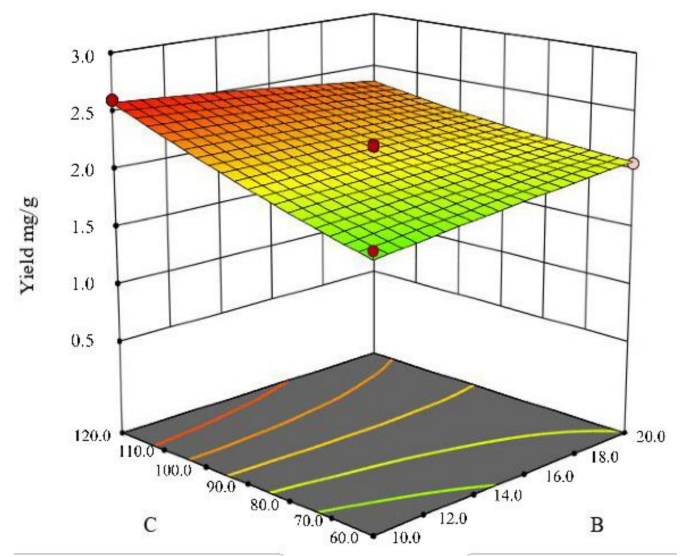

(c)

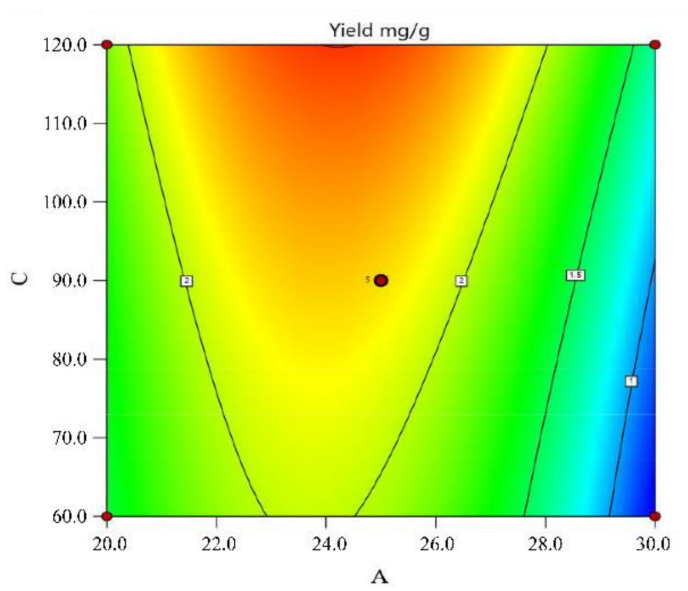

(b)

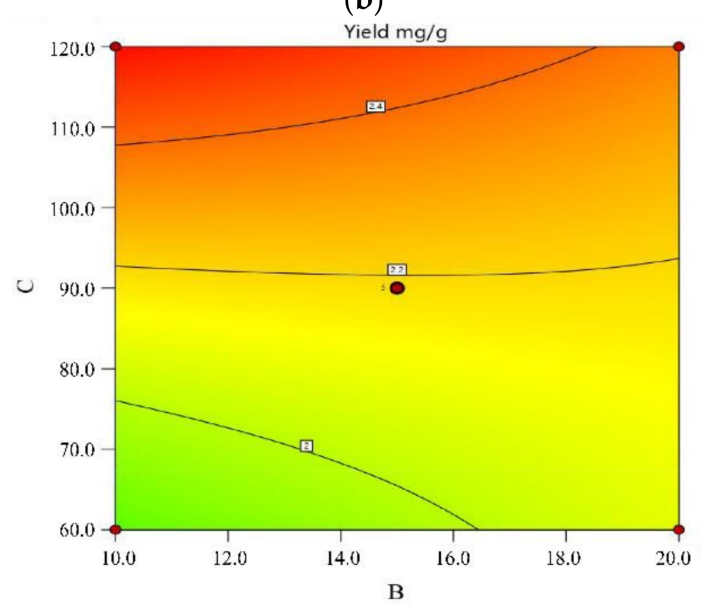

(d)

Figure 5. The 3D surfaces $(\mathbf{a}, \mathbf{c})$ and contour graphs $(\mathbf{b}, \mathbf{d})$ of the effects of ammonia concentration (A), solid-liquid ratio (B), and soaking time $(\mathrm{C})$ on the yield of astragaloside IV.

Figure $5 c$ shows the interaction between the solid-liquid ratio and soaking time. As the solid-liquid ratio was on the increase, the yield of saponins decreased. A higher solid-liquid ratio provided more opportunities for astragalus saponins and ammonia molecules, which could cause the destruction of saponins. As a phenomenon in flavonoids, glycosides could also produce aminoglycosides or aglycones as a result of alkali hydrolysis. The structure of those saponins could be undermined by alkali to varying degrees. As the soaking time was extended, the transformation from astragalosides replaced with an acetyl group to astragaloside IV was enhanced. The yield of the astragaloside IV significantly increased [15]. As shown in Figure 5c,d, the yield of astragaloside IV reached its maximum when the solid-liquid ratio and soaking time were approximately 10 and $120 \mathrm{~min}$, respectively.

\subsubsection{Optimization and Validation Procedures}

According to the constructed mode, the conditions were determined as follows. The ammonia concentration was $24.15 \%$, the solid-liquid ratio was $1: 10$, the soaking time was $120 \mathrm{~min}$, and the extraction time was $52 \mathrm{~min}$. In this circumstance, the theoretical content of astragaloside IV was predicted to be $2.635 \mathrm{mg} / \mathrm{g}$. After careful consideration, the ammonia concentration was set to $24 \%$, the solid-liquid ratio was set to $1: 10$, the soaking time was set to $120 \mathrm{~min}$, and the extraction time was set to $52 \mathrm{~min}$. The experiment was repeated three times to verify the formulation. The average content of astragaloside IV was $2.621 \pm 0.019 \mathrm{mg} / \mathrm{g}$, which was basically consistent with the values as predicted using 
the constructed model and comparable with the extraction efficiency achieved by Yan, Mingming et al. [30].

\subsection{The Changes in the Astragaloside IV Retention Rate Under Different Sterilization Conditions}

Since the structure of astragaloside IV may be affected in an alkaline environment [15], the process of thermal sterilization could further accelerate this process, thus resulting in a significant decrease in the yield of astragaloside IV. As suggested by the results shown in Figure 6, the retention rate of astragaloside IV in the solutions at varying $\mathrm{pH}$ values was determined by different sterilization conditions. The solutions with $\mathrm{pH}$ values of 3.0, 5.0, 7.0, and 9.0 represented acidic $(\mathrm{pH}<4.5)$, low-acidic $(4.5<\mathrm{pH}<7)$, neutral $(\mathrm{pH}=7)$, and alkaline $(\mathrm{pH}>7)$ food systems, respectively. As shown in Figure 6a, the thermal sterilization temperature was $85^{\circ} \mathrm{C}$. Astragaloside retention was weakened as the time of heat sterilization was extended. More specifically, the solutions with $\mathrm{pH}$ values of 3.0, 5.0, and 7.0 remained unchanged after $40 \mathrm{~min}$ of heating and showed a slight decrease $(97.19 \pm 1.91 \% \sim 98.45 \pm 1.40 \%)$ after $50-60 \mathrm{~min}$ of heating. The solution with a $\mathrm{pH}$ value of 9.0 dropped to $91.33 \pm 1.53 \%$ when heated for $30 \mathrm{~min}$. As heat sterilization was prolonged, the retention rate of astragaloside IV first declined to speed up and then slowed down to $74.83 \pm 1.61 \%$ in $60 \mathrm{~min}$, as shown in Figure $6 \mathrm{~b}$. The variation in Figure $6 \mathrm{c}$ is similar to that in Figure 6a, with the overall retention rate of astragaloside IV showing a decreasing trend. As shown in Figure $6 \mathrm{~b}$, the temperature was $90{ }^{\circ} \mathrm{C}$. After $60 \mathrm{~min}$ of heating, the retention of astragaloside IV in solutions with varying $\mathrm{pH}$ values was $91.83 \pm 1.01 \%$ (pH 3.0), $90.47 \pm 2.17 \%$ (pH 5.0), $92.53 \pm 0.74 \%$ (pH 7.0), and $64.67 \pm 1.53 \%$ (pH 9.0). The temperature was $95^{\circ} \mathrm{C}$ in Figure $6 \mathrm{c}$. When the sterilization time was $60 \mathrm{~min}$, the retention of astragaloside IV in solutions with varying $\mathrm{pH}$ values was $91.01 \pm 2.32 \%$ (pH 3.0), $88.80 \pm 2.21 \%(\mathrm{pH} 5.0), 89.57 \pm 1.91 \%(\mathrm{pH} 7.0)$, and $58.37 \pm 1.10 \%(\mathrm{pH} 9.0)$ For Figure $6 \mathrm{~d}$, the process at a high temperature and with a short sterilization time was performed. After heating $100^{\circ} \mathrm{C} / 15 \mathrm{~min}, 105^{\circ} \mathrm{C} / 15 \mathrm{~min}$, and $120^{\circ} \mathrm{C} / 15 \mathrm{~min}$, there was no significant difference $(p>0.05)$ between the retention rate of astragaloside IV in different $\mathrm{pH}$. In contrast, the retention rate of astragaloside IV at $\mathrm{pH} 9.0$ was reduced significantly. After being heated at $120{ }^{\circ} \mathrm{C}$ for $10 \mathrm{~min}$, however, the retention rate of astragaloside IV was $86.67 \pm 2.08 \%$, which was sufficient to preserve astragaloside IV in the alkaline solution better than in other germicidal conditions. In the meantime, astragaloside IV is the final form of transformation for astragalus saponins [13]. After heat sterilization, the astragaloside IV extracted by the alkaline solution could not be converted back to other saponins. In the solution, there were no other astragalus saponins detected (astragaloside I and astragaloside II).

In this paper, the stability of astragaloside in the process of thermal sterilization was first studied. Regarding Chinese herbal medicines (CHMs), it is common for the transformation of chemical constituents to occur as a result of high temperature, high humidity, and either high or low $\mathrm{pH}$ values. In this paper, astragaloside IV was extracted using an alkaline solution, and the acidity of the subsequent processing environment could vary according to the actual situation. Due to the potential of transformation between astragalus saponins, the stability of astragaloside is worth paying particular attention to. Up to now, however, there have been few studies focusing on the stability of astragalus saponins in different acidity levels at room temperature, and saponins lack stability in the process of thermal sterilization. For example, Mei X.D. et al. [15] made an attempt to extract different bases, but they ignored the subsequent stability. Additionally, no consideration was given to the potential damage caused by temperature to astragaloside IV. According to these experimental results, a higher sterilization temperature and a longer sterilization time were more damaging to astragaloside IV. Moreover, astragaloside IV can maintain stability and is also more resistant to heat sterilization in acidic, low-acidic, or neutral solution environments than in an alkaline solution, which is conducive to food processing. 


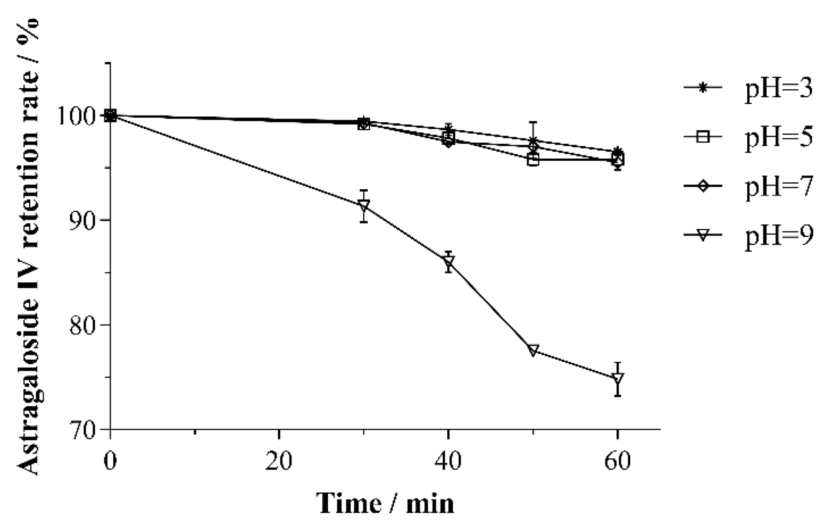

(a)

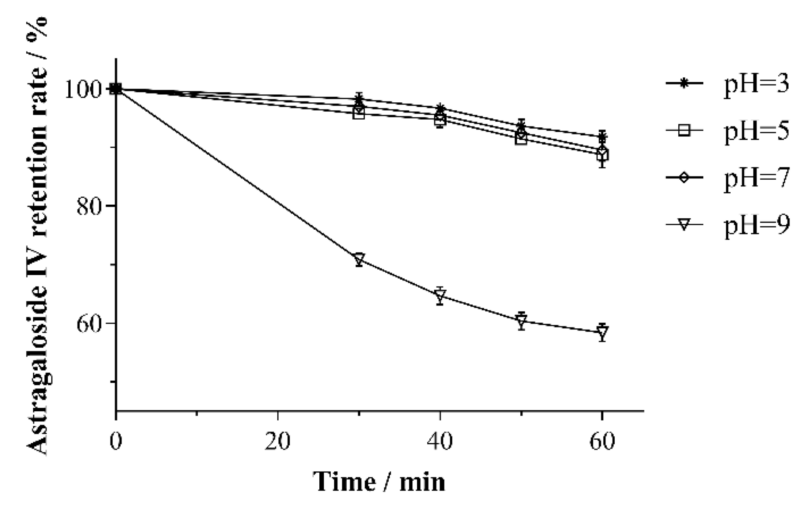

(c)

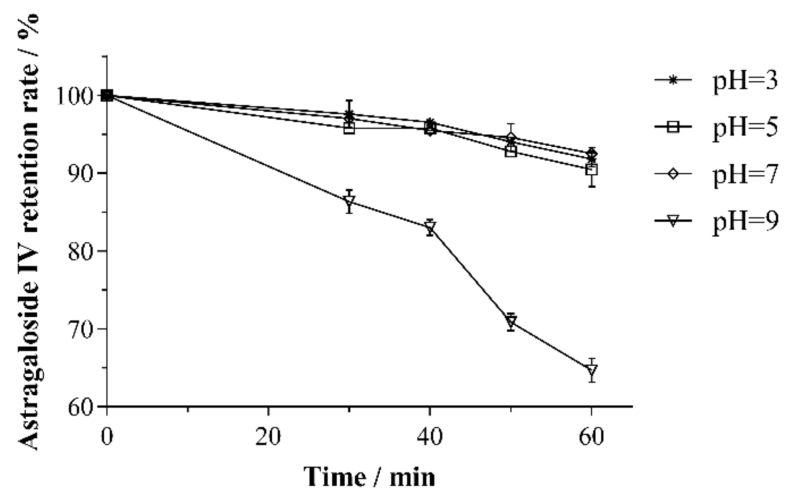

(b)

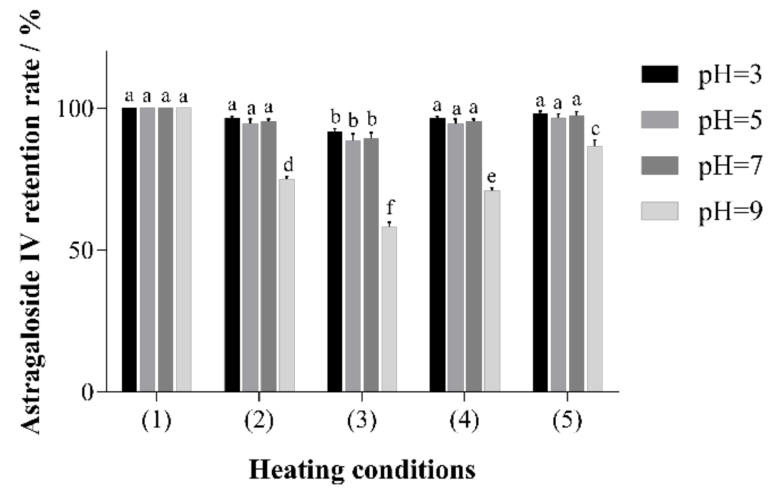

(d)

Figure 6. Changes in the retention rate of astragaloside IV of solutions under different sterilization conditions with different $\mathrm{pH}$ values. (a) The retention rate of astragaloside IV with different heating times at $85^{\circ} \mathrm{C}$. (b) The change of the retention rate of astragaloside IV with different heating times at $90^{\circ} \mathrm{C}$. (c) The change of the retention rate of astragaloside IV with different heating times at $95^{\circ} \mathrm{C}$. (d) The change of the retention rate of astragaloside IV with different heating conditions (group 1) at $100{ }^{\circ} \mathrm{C}$ for $0 \mathrm{~min}$, heating at $100^{\circ} \mathrm{C}$ for $15 \mathrm{~min}$ (group 2), heating at $100{ }^{\circ} \mathrm{C}$ for $30 \mathrm{~min}$ (group 3), heating at $105^{\circ} \mathrm{C}$ for $15 \mathrm{~min}$ (group 4), and heating at $120^{\circ} \mathrm{C}$ for $10 \mathrm{~min}$ (group 5). Different lowercase letter means differ significantly $(p<0.05)$.

\subsection{Changes in the Astragaloside IV Retention Rate under Different Storage Conditions}

For example, after $40 \mathrm{~min}$ of heating at $85^{\circ} \mathrm{C}$, the retention rate of astragaloside IV in the solution with different $\mathrm{pH}$ values $(\mathrm{pH} 3.0,5.0,7.0$, and 9.0) varied within 60 days of storage at room temperature. As shown in Figure $7 \mathrm{a}-\mathrm{c}$, the retention rate of astragaloside IV in acidic ( $\mathrm{pH} 3.0)$, low-acidic ( $\mathrm{pH} 5.0)$, and neutral ( $\mathrm{pH} 7.0)$ solutions decreased in the first 30 days of storage to $92.58 \pm 3.75 \%, 93.33 \pm 3.50 \%$, and $95.92 \pm 1.52 \%$, respectively. In contrast, the value dropped slightly to $92.98 \pm 1.97 \%, 93.33 \pm 1.50 \%$, and $94.50 \pm 0.91 \%$, respectively during 30-60 days. From Figure 7d, it can be seen that the yield of astragaloside IV in the alkaline ( $\mathrm{pH} 9.0)$ solution increased at a slow pace during storage and reached $104.88 \pm 1.46 \%$ after 60 days of storage. Since astragaloside IV is the basic skeleton structure of the astragaloside, it is a common transformation product for other astragalosides such as astragaloside I, astragaloside II, isoastragaloside II, astragaloside I, isoastragaloside I, acetylastragaloside I, and malonylastragaloside I [15]. Additionally, Figure 7 reveals no significant difference in the effect caused by cold storage and room temperature storage on astragaloside. In this study, the optimal extraction process was conducted to obtain an astragaloside IV extract. After thermal sterilization, the stability of astragaloside IV was assessed. Different from the work Chu C. et al. [13], an astragaloside standard was 
applied, and several Radix Astragali medicinal materials were taken as raw materials. After extraction was performed at room temperature, adjustment was made to the acidity of the solution to determine the change in astragaloside IV content under acidic, neutral, and alkaline environments. This was mainly attributed to the failure in completing the transformation of astragalus saponins in the early stage. Furthermore, Zheng N. et al. [16] conducted a study to demonstrate that astragaloside I is more susceptible to temperature and acidity than astragaloside I, thus confirming the above conjecture. Regarding food processing, the appropriate storage conditions can be determined according to the need to ensure product safety.

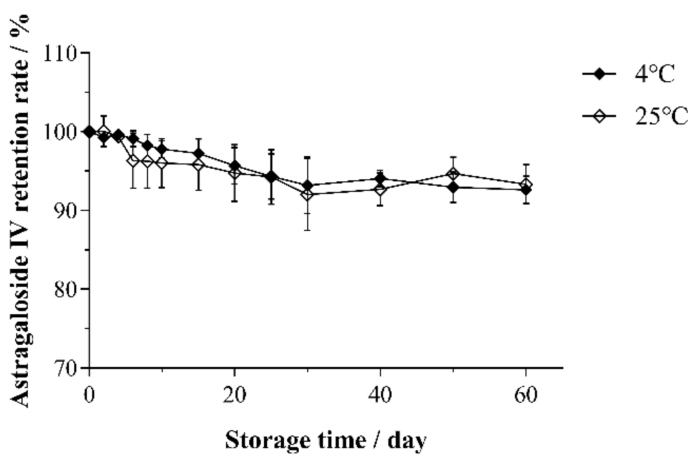

(a)

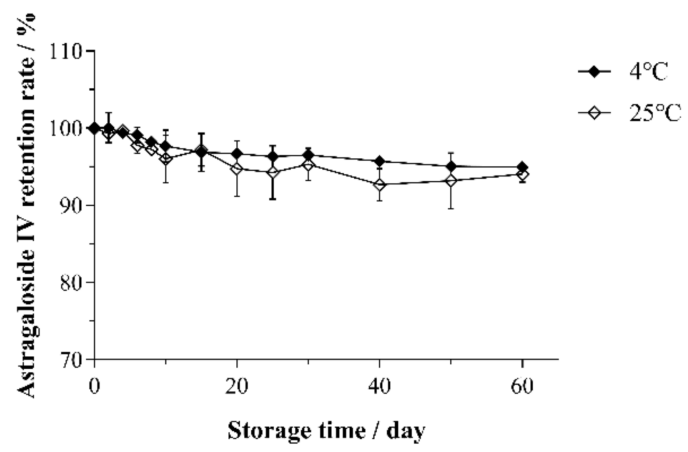

(c)

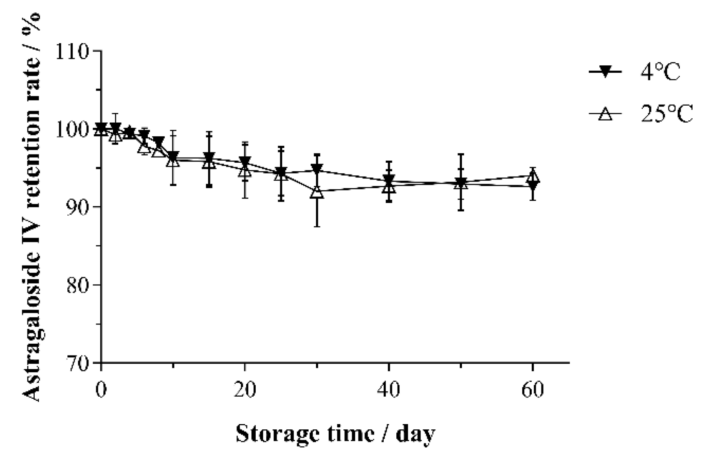

(b)

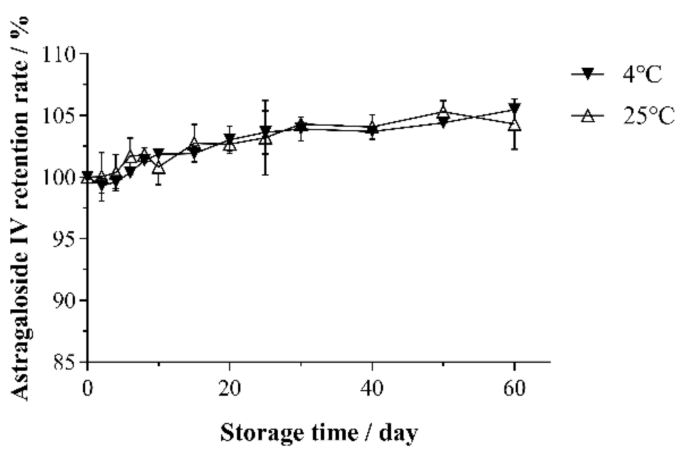

(d)

Figure 7. Changes in the retention rate of astragaloside IV in solutions with different $\mathrm{pH}$ values: (a) $\mathrm{pH} 3.0$, (b) $\mathrm{pH} 5.0$, (c) $\mathrm{pH} 7.0$, and (d) $\mathrm{pH} 9.0$ during the storage at 4 or $25^{\circ} \mathrm{C}$.

\section{Conclusions}

In recent years, Radix Astragali has been increasingly used in food processing, which makes it necessary to improve the yield of astragaloside IV during extraction. In this study, it was found out that both astragaloside I and astragaloside II were transformed completely to astragaloside IV under the optimal conditions. These conditions could be used to replace the traditional organic solvent reflux extraction method and are applicable in food processing because of their efficiency, low energy consumption, and non-toxicity. It was also found out that astragaloside IV was more stable in acidic, low-acidic, and neutral foods than in alkaline foods during sterilization. In alkaline foods, a high temperature and a short sterilization time can enhance the stability of astragaloside IV. Moreover, for acidic, lowacidic, and neutral foods, more thermal sterilization conditions can be selected. Moreover, it was discovered that the astragaloside IV obtained under the extraction conditions applied in this study could maintain a high stability during storage and facilitate food processing. In food processing, sterilization conditions can be determined on the basis of the experimental results obtained this study. For our future study, the focus will be placed on the changes in 
astragaloside IV during food processing for adaptation to more food processing scenarios, and physiological function will be analyzed.

Author Contributions: Conceptualization, L.X. and L.Z.; funding acquisition, L.Z.; methodology, L.X. and L.Z.; writing-original draft, L.X.; writing-review and editing, K.W. and J.J. All authors will be informed about each step of manuscript processing including submission, revision, revision reminder, etc. via emails from our system or assigned Assistant Editor. All authors have read and agreed to the published version of the manuscript.

Funding: This research was funded by National Key Technology R\&D Program for the 13th five-year plan, grant number $2017 \mathrm{BAD} 33 \mathrm{~B} 05$ and the Jiangsu province "Collaborative Innovation Center for Food Safety and Quality Control".

Conflicts of Interest: The authors declare no conflict of interest. The funders had no role in the design of the study; in the collection, analyses, or interpretation of data; in the writing of the manuscript; or in the decision to publish the results.

Sample Availability: Samples of the astragaloside I, astragaloside II, and astragaloside IV standard compounds are available from the authors.

\section{Appendix A}

Table A1. The experiment results of response surface methodology. Data were expressed as mean \pm standard deviation (SD) $(n=3)$.

\begin{tabular}{|c|c|c|c|c|c|c|c|}
\hline $\begin{array}{l}\text { Treatment } \\
\text { Number }\end{array}$ & $\begin{array}{c}\text { Ammonia } \\
\text { Concentration } \\
\text { (A) }(\%, v / v)\end{array}$ & $\begin{array}{c}\text { Solid- } \\
\text { Liquid Ratio } \\
\text { (B) }(\mathrm{mL} / \mathrm{g})\end{array}$ & $\begin{array}{l}\text { Soaking } \\
\text { Time (C) } \\
\text { (min) }\end{array}$ & $\begin{array}{l}\text { Extraction } \\
\text { Time (D) } \\
\text { (min) }\end{array}$ & $\begin{array}{l}\text { Content of } \\
\text { Astragaloside } \\
\text { IV (mg/g) }\end{array}$ & $\begin{array}{l}\text { Astragaloside } \\
\text { I (mg/g) }\end{array}$ & $\begin{array}{l}\text { Astragaloside } \\
\text { II (mg/g) }\end{array}$ \\
\hline 1 & 25 & 10 & 90 & 0 & $2.012 \pm 0.073$ & Not detected & Not detected \\
\hline 2 & 25 & 20 & 120 & 30 & $2.301 \pm 0.011$ & Not detected & Not detected \\
\hline 3 & 25 & 10 & 60 & 30 & $1.904 \pm 0.023$ & Not detected & Not detected \\
\hline 4 & 25 & 15 & 120 & 0 & $2.235 \pm 0.084$ & Not detected & Not detected \\
\hline 5 & 30 & 15 & 60 & 30 & $0.701 \pm 0.036$ & $0.107 \pm 0.005$ & $0.142 \pm 0.010$ \\
\hline 6 & 25 & 20 & 90 & 0 & $2.049 \pm 0.042$ & Not detected & Not detected \\
\hline 7 & 25 & 20 & 90 & 60 & $2.283 \pm 0.086$ & Not detected & Not detected \\
\hline 8 & 25 & 15 & 90 & 30 & $2.215 \pm 0.061$ & Not detected & Not detected \\
\hline 9 & 30 & 15 & 120 & 30 & $1.436 \pm 0.021$ & Not detected & $0.086 \pm 0.002$ \\
\hline 10 & 30 & 20 & 90 & 30 & $0.938 \pm 0.025$ & $0.067 \pm 0.004$ & $0.103 \pm 0.003$ \\
\hline 11 & 20 & 15 & 60 & 30 & $1.473 \pm 0.077$ & Not detected & $0.079 \pm 0.002$ \\
\hline 12 & 25 & 15 & 90 & 30 & $2.157 \pm 0.113$ & Not detected & Not detected \\
\hline 13 & 30 & 15 & 90 & 0 & $0.851 \pm 0.032$ & $0.076 \pm 0.003$ & $0.129 \pm 0.004$ \\
\hline 14 & 30 & 10 & 90 & 30 & $0.940 \pm 0.023$ & $0.071 \pm 0.002$ & $0.116 \pm 0.003$ \\
\hline 15 & 25 & 15 & 60 & 60 & $1.981 \pm 0.049$ & Not detected & Not detected \\
\hline 16 & 20 & 20 & 90 & 30 & $1.702 \pm 0.026$ & Not detected & Not detected \\
\hline 17 & 25 & 20 & 60 & 30 & $2.063 \pm 0.036$ & Not detected & Not detected \\
\hline 18 & 25 & 10 & 120 & 30 & $2.602 \pm 0.072$ & Not detected & Not detected \\
\hline 19 & 25 & 15 & 90 & 30 & $2.151 \pm 0.034$ & Not detected & Not detected \\
\hline 20 & 25 & 15 & 90 & 30 & $2.220 \pm 0.065$ & Not detected & Not detected \\
\hline 21 & 20 & 15 & 90 & 0 & $1.566 \pm 0.073$ & Not detected & $0.067 \pm 0.002$ \\
\hline 22 & 20 & 15 & 90 & 60 & $1.757 \pm 0.029$ & Not detected & Not detected \\
\hline 23 & 20 & 15 & 120 & 30 & $1.861 \pm 0.016$ & Not detected & Not detected \\
\hline 24 & 25 & 15 & 90 & 30 & $2.205 \pm 0.035$ & Not detected & Not detected \\
\hline 25 & 25 & 10 & 90 & 60 & $2.102 \pm 0.039$ & Not detected & Not detected \\
\hline 26 & 30 & 15 & 90 & 60 & $0.882 \pm 0.026$ & $0.069 \pm 0.003$ & $0.125 \pm 0.002$ \\
\hline 27 & 25 & 15 & 120 & 60 & $2.590 \pm 0.108$ & Not detected & Not detected \\
\hline 28 & 20 & 10 & 90 & 30 & $1.660 \pm 0.025$ & Not detected & $0.062 \pm 0.002$ \\
\hline 29 & 25 & 15 & 60 & 0 & $1.801 \pm 0.049$ & Not detected & Not detected \\
\hline
\end{tabular}




\section{References}

1. Joung, J.Y.; Lee, J.S.; Cho, J.H.; Lee, D.S.; Ahn, Y.C.; Son, C. The Efficacy and Safety of Myelophil, an Ethanol Extract Mixture of Astragali Radix and Salviae Radix, for Chronic Fatigue Syndrome: A Randomized Clinical Trial. Front. Pharmacol. 2019, 10, 991-1003. [CrossRef]

2. Turck, D.; Castenmiller, J.; De Henauw, S.; Hirsch-Ernst, K.I.; Kearney, J.; Maciuk, A. Safety of a botanical extract derived from Panax notoginseng and Astragalus membranaceus (AstraGin (TM)) as a novel food pursuant to Regulation (EU) 2015/2283. Efsa J. 2020, 18, 1-16.

3. Butkute, B.; Benetis, R.; Padarauskas, A.; Ceseviciene, J.; Dagilyte, A.; Taujenis, L.; Rodovicius, H.; Lemeziene, N.; Lemeziene, N. Young herbaceous legumes-a natural reserve of bioactive compounds and antioxidants for healthy food and supplements. $J$. Appl. Botany Food Q./Angewandte Botanik 2017, 90, 346-353.

4. Korany, M.A.; Moneeb, M.S.; Asaad, A.M.; El-Sebakhy, N.A.; El-Banna, A.A. Analysis of astragalosides I, II and IV in some Egyptian Astragalus species and Astragalus dietary supplements using high-performance liquid chromatography/evaporative light scattering detector and non-parametric regression. Phytochem. Anal. 2020, 31, 594-605. [CrossRef] [PubMed]

5. Wenjing, Z.; Biwang, L.; Meng, Z.; Shanshan, W.; Nan, Q. Preparation technology and efficacy of the anti-haze Qingfei drink. Sci. Technol. Food Ind. 2020, 15, 43-52.

6. Guo, Z.; Lou, Y.; Kong, M.; Luo, Q.; Liu, Z.; Wu, J. A Systematic Review of Phytochemistry, Pharmacology and Pharmacokinetics on Astragali Radix: Implications for Astragali Radix as a Personalized Medicine. Int. J. Mol. Sci. 2019, 20, 1463. [CrossRef]

7. Graziani, V.; Scognamiglio, M.; Esposito, A.; Fiorentino, A.; D'Abrosca, B. Chemical diversity and biological activities of the saponins isolated from Astragalus genus: Focus on Astragaloside IV. Phytochem. Rev. 2019, 1133-1166. [CrossRef]

8. Qi, Y.; Gao, F.; Hou, L.; Wan, C. Anti-Inflammatory and Immunostimulatory Activities of Astragalosides. Am. J. Chin. Med. 2017, 45, 1157-1167. [CrossRef] [PubMed]

9. Jiang, K.; Lu, Q.; Li, Q.; Ji, Y.; Chen, W.; Xue, X. Astragaloside IV inhibits breast cancer cell invasion by suppressing Vav3 mediated Rac1/MAPK signaling. Int. Immunopharmacol. 2017, 42, 195-202. [CrossRef]

10. Chan, W.S.; Durairajan, S.S.K.; Lu, J.H.; Wang, Y.; Xie, L.X.; Kum, W.F.; Koo, I.; Yung, K.K.L.; Li, M. Neuroprotective effects of Astragaloside IV in 6-hydroxydopamine-treated primary nigral cell culture. Neurochem. Int. 2009, 55, 414-422. [CrossRef]

11. Yang, H.; Zhao, Z.; Gao, S.; Lei, J.; Jiang, Y. Extraction of astragaloside in Astragali broken-cell pieces by accelerated solvent extraction. Lishizhen Medicine and Materia Medica Research 2017, 28, 310-312.

12. Liu, M.; Zhou, J.; Zhang, Q.-W.; Liu, F. Amic solution hydrolysis for improving content of astragaloside IV in extract of Radix Astragali. China J. Chin. Mater. Medica 2008, 33, 635-643.

13. Chu, C.; Liu, E.H.; Qi, L.W.; Li, P. Transformation of astragalosides from Radix Astragali under acidic, neutral, and alkaline extraction conditions monitored by LC-ESI-TOF/MS. Chin. J. Nat. Med. 2014, 12, 314-320. [CrossRef]

14. Qi, L.-W.; Cao, J.; Li, P.; Wang, Y.-X. Rapid and sensitive quantitation of major constituents in Danggui Buxue Tang by ultra-fast HPLC-TOF/MS. J. Pharm. Biomed. Anal. 2009, 49, 502-507. [CrossRef] [PubMed]

15. Mei, X.; Wang, Y.; Liu, Z.; Wang, S.; Dong, F.; Wang, Z.; Qiao, Y.; Zhang, J. The chemical transformations for Radix Astragali via different alkaline wash conditions by quantitative and qualitative analyses. J. Pharm. Biomed. Anal. 2020, 185, 113164. [CrossRef]

16. Zheng, N.; Shi, Y.; Ji, Y.; Lu, W. Stability and transformation of astragaloside IV, III and I. Cent. S Pharm. 2014, 22, $1062-1065$.

17. Feng, X.; Huang, K.; Wei, G.; Huang, Y. Changes of Astragalosides and Astragaloside IV Contents in Acid and Alkali Solution. Trad. Chin. Drug Res. Clin. Plarmacol. 2010, 21, 533-537.

18. Linhares, M.D.F.D.; Filho, E.G.A.; Silva, L.M.A.; Fonteles, T.V.; Wurlitzer, N.J.; de Brito, E.S.; Fernandes, F.A.; Rodrigues, S. Thermal and non-thermal processing effect on açai juice composition. Food Res. Int. 2020, 136, 109506. [CrossRef] [PubMed]

19. Hu, Y.; Cui, X.; Zhang, Z.; Chen, L.; Zhang, Y.; Wang, C.; Yang, X.; Qu, Y.; Xiong, Y. Optimisation of Ethanol-Reflux Extraction of Saponins from Steamed Panax notoginseng by Response Surface Methodology and Evaluation of Hematopoiesis Effect. Molecules 2018, 23, 1206. [CrossRef]

20. Fang, J.-L.; Luo, Y.; Yuan, K.; Guo, Y.; Jin, S.-H. Preparation and evaluation of an encapsulated anthocyanin complex for enhancing the stability of anthocyanin. LWT 2020, 117, 108543. [CrossRef]

21. Du, K.; Li, J.; Guo, X.; Li, Y.; Chang, Y. New Method for Determination of Astragaloside IV in Astragali Radix. Chin. J. Exp. Trad. Med. Formulae 2020, 26, 132-137.

22. Shaw, L.-H.; Lin, L.-C.; Tsai, T.-H. HPLC-MS/MS Analysis of a Traditional Chinese Medical Formulation of Bu-Yang-Huan-WuTang and Its Pharmacokinetics after Oral Administration to Rats. PLoS ONE 2012, 7, e43848. [CrossRef] [PubMed]

23. Tamjidi, F.; Shahedi, M.; Varshosaz, J.; Nasirpour, A. Stability of astaxanthin-loaded nanostructured lipid carriers as affected by $\mathrm{pH}$, ionic strength, heat treatment, simulated gastric juice and freeze-thawing. J. Food Sci. Technol. 2017, 54, 3132-3141. [CrossRef]

24. Ghavi, P.P. The extraction process optimization of antioxidant polysaccharides from Marshmallow (Althaea officinalis L.) roots. Int. J. Biol. Macromol. 2015, 75, 51-57. [CrossRef]

25. Chen, Y.; Xie, M.-Y.; Gong, X.-F. Microwave-assisted extraction used for the isolation of total triterpenoid saponins from Ganoderma atrum. J. Food Eng. 2007, 81, 162-170. [CrossRef]

26. Vongsangnak, W.; Gua, J.; Chauvatcharin, S.; Zhong, J.-J. Towards efficient extraction of notoginseng saponins from cultured cells of Panax notoginseng. Biochem. Eng. J. 2004, 18, 115-120. [CrossRef] 
27. Du, K.-Z.; Li, J.; Guo, X.; Li, Y.; Chang, Y.-X. Quantitative Analysis of Phenolic Acids and Flavonoids in Cuscuta chinensis Lam. by Synchronous Ultrasonic-Assisted Extraction with Response Surface Methodology. J. Anal. Methods Chem. 2018, $2018,1-10$. [CrossRef]

28. Zhang, X.-F.; Yang, J.-L.; Chen, J.; Shi, Y.-P. Optimization of a Decoction Process for an Herbal Formula Using a Response Surface Methodology. J. AOAC Int. 2017, 100, 1776-1784. [CrossRef] [PubMed]

29. Wu, M.; Wang, M.; Zhang, Y.; Zhou, J.; Xu, L.; Xia, G.; Yan, Z.; Shen, Y.; Yang, H. Efficient and clean preparation of rare prosaikogenin D by enzymatic hydrolysis of saikosaponin B2 and response surface methodology optimization. Enzym. Microb. Technol. 2020, 142, 109690. [CrossRef]

30. Yan, M.-M.; Liu, W.; Fu, Y.-J.; Zu, Y.-G.; Chen, C.-Y.; Luo, M. Optimisation of the microwave-assisted extraction process for four main astragalosides in Radix Astragali. Food Chem. 2010, 119, 1663-1670. [CrossRef] 